\title{
A large disordered region confers a wide spanning volume to vertebrate Suppressor of Fused as shown in a trans-species solution study.
}

\section{Staëlle Makamte', Amira Jabrani' ${ }^{1}$, Annick Paquelin ${ }^{1}$, Anne Plessis ${ }^{2}$, Mathieu Sanial ${ }^{2}$, Aurélien Thureau $^{3}$, Olga Rudenko ${ }^{3}$, Francesco Oteri ${ }^{4,5}$, Marc Baaden ${ }^{4}$, Valérie Biou ${ }^{1}$}

1 Laboratoire de Biologie Physico-Chimique des Protéines Membranaires, Université de Paris, UMR 7099 CNRS, Institut de Biologie Physico-Chimique, 13 rue Pierre et Marie Curie, 75005 Paris, France. 2 Université de Paris, CNRS, Institut Jacques Monod, F-75006 Paris, France. 3 Synchrotron SOLEIL, F-91192 Gif sur Yvette, France. 4 Laboratoire de Biochimie Théorique, UPR 9080 CNRS, Institut de Biologie Physico-Chimique, 13 rue Pierre et Marie Curie, 75005 Paris, France. 5, FO’s present address: Laboratoire de Biologie Computationnelle et Quantitative UMR7238 CNRS, Sorbonne Université, Institut de Biologie Paris-Seine, Bât. C, 4 place Jussieu, 75005 Paris, France.

Running title: SuFu disordered region is disclosed by solution studies

Keywords: Hedgehog signal; intrinsically disordered protein; small-angle X-ray scattering

Corresponding author: Valerie Biou valerie.biou@ibpc.fr 


\begin{abstract}
Hedgehog $(\mathrm{Hh})$ pathway inhibition by the conserved protein Suppressor of Fused $(\mathrm{SuFu})$ is crucial to vertebrate development. By constrast, SuFu removal has little effect in drosophila.

Previous publications showed that the crystal structures of human and drosophila SuFu consist of two ordered domains that are capable of breathing motions upon ligand binding. However, the crystal structure of human SuFu does not give information about $20 \mathrm{~N}$-terminal residues (IDR1) and an eightyresidue-long disordered region (IDR2) in the C-terminus, whose function is important for the pathway repression. These two IDRs are species-dependent.

We studied SuFu's structure in solution, both with circular dichroism and small angle X-ray scattering, comparing drosophila, zebrafish and human species, to better understand this considerable difference. Our studies show that, in spite of similar crystal structures restricted to ordered domains, drosophila and vertebrate $\mathrm{SuFu}$ have very different structures in solution. The IDR2 of vertebrates spans a large area, thus enabling it to reach for partners and be accessible for post-translational modifications. Furthermore, we show that the IDR2 region is highly conserved within phyla but varies in length and sequence, with insects having a shorter disordered region while that of vertebrates is broad and mobile. This major variation may explain the different phenotypes observed upon SuFu removal.
\end{abstract}

\title{
Introduction
}

The conserved Hedgehog (Hh) signalling pathway is essential to specify cell and tissue fate during embryogenesis and later to regulate stem cell homeostasis (Jiang and Hui, 2008). Discovered in drosophila, where it ensures proper polarisation of the larva segments (Nusslein-Volhard and Wieschaus, 1980), the Hh pathway has since been identified in many animals where it controls multiple patterning events, and has rapidly emerged as a key player in oncogenesis by controlling tumour promotion or/and progression (Pak and Segal, 2016). In the absence of Hh signal the G-Protein Coupled Receptor-related Smoothened (Smo) activity is inhibited by the Hh receptor Patched (Ptc). Binding of Hh releases this negative effect, triggering the activation of Smo, probably by favouring its association with intramembrane cholesterol (for review see (Hu and Song, 2019)). Smo activation also involves its relocalisation from intracellular vesicles to the plasma membrane in fly, and its rapid translocation from the base to the tip of the primary cilium in mammals (Hooper and Scott, 1989; Ingham et al., 1991; 
Marigo et al., 1996; Zhang et al., 2018). Via its interactions with a cytoplasmic protein complex, Smo activates kinases that change the phosphorylation state of several proteins of this complex and remodel their interactions. As a result, the transcription factor(s) of the Ci/Gli family are activated and transported into the nucleus where they in turn activate the expression of specific target genes, thus changing the cell fate. The molecular backbone of the pathway is conserved but it becomes more complex from flies to mammals. For example in vertebrates, three transcription factors are involved: Gli 1, 2 and 3; their activation takes place at the primary cilium and depends on intraflagellar trafficking and results in their translocation into the nucleus (Huangfu et al., 2003).

The proper function of the Hh pathway at the right time and place is so crucial that several levels of negative regulation exist to prevent inappropriate Hh activation. This includes the products of two tumour suppressor genes: Ptc at the cell surface and Suppressor of Fused ( $\mathrm{SuFu}$ ) inside the cell. Removal of $\mathrm{SuFu}$ has very different consequences in drosophila and mammals. In drosophila, the phenotype of sufu removal is only visible in the context of the absence of fused kinase (Preat, 1992). Conversely, SuFu $\mathrm{KO}$ mice die in utero and exhibit heart, brain and CNS defects due to a Hh constitutional activation (Cooper et al., 2005; Svard et al., 2006).

Discovered in drosophila (Preat, 1992), where it interacts with a microtubule-associated complex including kinase Fused (Monnier et al., 1998), SuFu is a $55 \mathrm{kDa}$ protein that plays an inhibitory role via the sequestration of full-length $\mathrm{Ci}$ in the cytoplasm. In response to $\mathrm{Hh}$, this negative effect of $\mathrm{SuFu}$ is suppressed by the kinase Fused. SuFu then accompanies Ci into the nucleus (Sisson et al., 2006). In zebrafish, SuFu regulates Gli transfer to the primary cilium (Maurya et al., 2013) and SuFu morpholino knock down affects eye, ear and muscle development, identifying SuFu as a medium-level inhibitor of the Hh pathway (Koudijs et al., 2005). In mammals, SuFu is essential for the proper differentiation of many cell types including neural cells (Yabut et al., 2015) and it plays a role in white/brown adipocyte determination and obesity (Pospisilik et al., 2010). Here also, SuFu acts upstream of the Gli transcription factors, controlling their fate. It was thus shown to sequester full-length Gli 1 and 2 transcription factors in the cytoplasm in the absence of Hh. Moreover, SuFu also plays nuclear roles with the repressor forms of Gli as it was shown to recruit the SAP18-mSin3 repressor complex to the Gli binding sites (PacesFessy et al., 2004). Hh binding to Ptc represses this negative effect, allowing the Gli proteins accompanied by SuFu- to enter the nucleus. The present state of research describes $\mathrm{SuFu}$ as a hub, interacting with many partner proteins, and the interactions are remodelled when Hh is activated, mostly 
via post-transcriptional modifications, such as phosphorylation and ubiquitylation, of $\mathrm{SuFu}$ (Chen et al., 2011; Raducu et al., 2016; Wang et al., 2016).

Figure $\mathrm{S} 1$ shows an amino acid sequence alignment of $\mathrm{SuFu}$ from three species belonging to different phyla: Homo sapiens (hSuFu), Danio rerio (zebrafish) (zSuFu) and Drosophila melanogaster (dSuFu). The crystal structures of hSuFu and dSuFu (Cherry et al., 2013; Zhang et al., 2013) harbour two ordered domains corresponding to the regions framed in blue and pink boxes in the Figure S1, with respectively $41 \%$ and $24 \%$ identity between $\mathrm{hSuFu}, \mathrm{zSuFu}$ and $\mathrm{dSuFu}$. The $\mathrm{N}$-terminal domain is also found in prokaryotic proteins, amongst which antitoxin systems, another process involving protein-protein interactions (Zhang et al., 2011), and the C-terminal domain is specific to $\mathrm{SuFu}$ proteins. In addition to those two domains known from the crystal structures, Figure S1 shows that $\mathrm{SuFu}$ amino acid sequence contains two additional regions predicted to be intrinsically disordered by the PONDR server (http://www.pondr.com). The N-terminus disordered region IDR1 (in a dotted grey box) and the second region IDR2 (in a dotted black box) stemming from the C-terminal domains are the sequences that harbour the most striking differences between drosophila and vertebrates with $6 \%$ identical residues.

The N-terminal domain has a 7-stranded twisted beta sheet followed by a 3-helix bundle, and spans residues 30-263 (as numbered in hSuFu). The C-terminal domain has two beta sheets with 6 and 4 strands respectively and two helices; it consists of residues 267-280 and 356-457. The structure of free hSuFu (pdb code 4KM9/4BL8) is extended and the two domains become close to each other when a peptide from Gli is bound to $\mathrm{hSuFu}$ (pdb code $4 \mathrm{KMD} / 4 \mathrm{BLB}$ ), showing a potential tendency of the two domains to move with respect to each other. The drosophila $\mathrm{SuFu}$ crystal structure pdb code $4 \mathrm{KMA}$ spans residues 13-259 for the N-terminal domain and 265-454 for the C-terminal domain. Both domains have very similar folds to the human protein, but their relative orientations are different. The IDR2 region, which is disordered in the human structure, has only five disordered amino acids in the drosophila crystal structure. The rest of the sequence corresponding to IDR2 is structured and caps the C-terminal domain, but it has no regular secondary structure.

Due to the absence of structural information for full-length vertebrate $\mathrm{SuFu}$, we used circular dichroism (CD) and small angle X-ray scattering (SAXS) to investigate the solution structures of SuFu proteins from Drosophila melanogaster, Danio rerio and Homo sapiens. We also studied $\mathrm{hSuFu} \triangle 30$, a truncated mutant of hSuFu devoid of its IDR1 that allowed us to better analyse the effect of IDR2 alone. We found that the solution structure of the drosophila protein is very similar to the crystal structure, and 
investigated its breathing movements by normal mode analysis. Human and zebrafish SuFu show similar dimensions and exhibit an N-terminal IDR1 region that seems to remain close to the N-terminal domain, while the IDR2 loop in the C-terminal domain spans a large volume and can thus fetch its partner protein a long way from its core. Our present studies show that, even though their crystal structures are quite similar, the solution structures of vertebrate and drosophila $\mathrm{SuFu}$ are rather different and we discuss the specificity of IDR2 in the context of SuFu interaction with partner proteins.

\section{Experimental procedures}

\section{1) Protein expression and purification.}

$\mathrm{dSuFu}$ and hSuFu were cloned and expressed as previously described (Jabrani et al., 2017).

The cDNA encoding dSuFu was cloned in the pDEST17 (Invitrogen) expression vector according to standard Gateway ${ }^{\mathrm{TM}}$ protocols. The resulting plasmid encoded an $\mathrm{N}$-terminal hexahistidine tag and a TEV protease cleavage site before the gene of interest. E. coli strain C41(DE3) (Miroux and Walker, 1996) were grown in 2 YT medium at $37^{\circ} \mathrm{C}$ until the optical density reached 0.6 . Protein expression was induced with $0.2 \mathrm{mM}$ isopropyl $\beta$-thiogalactoside overnight at $20^{\circ} \mathrm{C}$. Bacterial cells were recovered by centrifugation at $5000 \mathrm{~g}$ for $15 \mathrm{~min}$ and frozen at $-80^{\circ} \mathrm{C}$. Similar procedures were used for the cDNAs encoding $\mathrm{hSuFu}$ and $\mathrm{zSuFu}$ respectively, which were cloned in a pACYDuet vector. $\mathrm{hSuFu} \triangle 30$ construct was produced by truncating the nucleotides coding for the first 30 aminoacids of $\mathrm{hSuFu}$. Cells were lysed in $50 \mathrm{mM}$ HEPES pH 7.5, $500 \mathrm{mM} \mathrm{NaCl}, 5 \mathrm{mM}$ imidazole, $10 \%$ glycerol using a Constant Cell disruptor at $2.3 \mathrm{kbar}$, then centrifuged for 1 hour at $9000 \mathrm{~g}$. The supernatant was loaded onto a Nickel affinity column (Roche), washed in the loading buffer and eluted in a buffer containing $200 \mathrm{mM}$ imidazole. The eluate was collected and the buffer exchanged against $10 \mathrm{mM}$ HEPES pH 7.5, $100 \mathrm{mM}$ $\mathrm{NaCl}, 10 \%$ glycerol, $2.5 \mathrm{mM} \mathrm{MgCl} 2$ and $2 \mathrm{mM} \beta$-mercaptoethanol on a concentrator. Then the protein solution was incubated with Tobacco Etch Virus protease for 24 hours at $10^{\circ} \mathrm{C}$ to cleave the 6-Histidine tag and the associated linker. The cleavage mixture was then dialysed back into the lysis buffer and incubated with Nickel affinity gel (Roche) for one hour to separate the cleaved protein from the TEV protease and tag. The flow through was then concentrated and injected on a Superdex 200 10/300 gel filtration column equilibrated with HEPES 50mM pH 7.5, $\mathrm{NaCl} 200 \mathrm{mM}$, glycerol 10\%, Dithiothreitol $5 \mathrm{mM}$ or bis-tris $50 \mathrm{mM} \mathrm{pH} 5.5, \mathrm{NaCl} 50 \mathrm{mM}$, glycerol 10\% and loaded onto a HiTrapQ column, washed 
and eluted in a $\mathrm{NaCl}$ gradient. The fractions corresponding to pure $\mathrm{SuFu}$ protein were concentrated and used for future studies.

CD studies were performed in the protein in two different buffers: A) HEPES HCl 50mM pH 7.5, $\mathrm{NaCl}$ 200mM, glycerol 10\%, Dithiothreitol 2mM or B) bis-tris $50 \mathrm{mM} \mathrm{pH} \mathrm{5.5,} \mathrm{NaCl} 200 \mathrm{mM}$, glycerol 10\%. SAXS studies were performed in buffer A only.

\section{2) SRCD measurements}

Synchrotron radiation circular dichroism (SRCD) was measured at the DISCO beamline (SOLEIL synchrotron, Gif sur Yvette, France). All sample concentrations were measured using the absorbance at $280 \mathrm{~nm}$, after centrifugation and just prior to measurement, using a theoretical extinction coefficient of $59400 \mathrm{M}^{-1} \cdot \mathrm{cm}^{-1}$ for $\mathrm{dSuFu}, 67775 \mathrm{M}^{-1} \cdot \mathrm{cm}^{-1}$ for $\mathrm{hSuFu}$ and $66390 \mathrm{M}^{-1} \cdot \mathrm{cm}^{-1}$ for $\mathrm{zSuFu}$. Concentrations were found to be between 4 and $10 \mathrm{mg} / \mathrm{ml}$.

The beamline monochromator was calibrated using a camphorsulfonic acid solution prior to measurements. One microlitre of sample was deposited on one face of a calcium fluoride circular cuvette (Hellma), then the second face was carefully positioned and the cuvette closed by capillary force. Interferometry measurement showed the optical path to be $2.3 \mu \mathrm{m}$. The cuvette was placed in an airtight, metal sample holder that was positioned in the beamline Peltier temperature-controlled chamber, allowing for quick temperature changes with very little evaporation. Spectra were measured between 280 and $170 \mathrm{~nm}$, each final spectrum being averaged from three repeated measurements. Thermal unfolding measurements were performed by averaging three spectra collected in steps of $5^{\circ} \mathrm{C}$ between 10 and $90^{\circ} \mathrm{C}$. Buffer spectra were taken in the same conditions for subtraction from the protein spectra. Spectra were then processed for buffer subtraction and scaling using the CDTools program (Lees et al., 2004). The melting temperature was evaluated by fitting the curve displaying the CD at $208 \mathrm{~nm}$ with a sigmoidal function in Kaleidagraph (Synergy Software), as a function of temperature.

\section{3) Small angle $X$-ray scattering measurements}

The SAXS measurements were done at the SWING beamline (Thureau et al., submitted.) at the SOLEIL synchrotron (Gif sur Yvette, France). The sample was injected at a flux of $0.3 \mathrm{ml} /$ minute into an Agilent Bio SEC-3-300 size exclusion column pre-equilibrated in the indicated buffer and mounted on an HPLC system, prior to its analysis in the X-ray beam. The X-ray energy was $12 \mathrm{keV}$. For dSuFu, measurements were carried out with an Aviex charge coupled device detector, the system being programmed to measure 100 images of the buffer then 240 images of the protein, using a 1-second exposure time interspaced by $500 \mathrm{~ms}$. For hSuFu, hSuFu $\Delta 30$ and zSuFu measurements, an Eiger 4M pixel detector was used. 600 
images were measured with $990 \mathrm{~ms}$ exposure and $10 \mathrm{~ms}$ dead time each. The sample to detector distance is listed in Table 2.

The images were processed using the FOXTROT software (https://www.synchrotronsoleil.fr/en/beamlines/swing) developed at the SOLEIL synchrotron. The curves derived from buffer measurement were averaged and subtracted from the buffer plus protein curves. Then the radius of gyration Rg was calculated using a Guinier plot on the most intense curve and extended to all scattering curves, thus producing a plot of $\mathrm{Rg}$ and scattered intensity, $\mathrm{I}_{0}$, for all images. Curves from $\mathrm{hSuFu}$ and zSuFu were processed with US-SOMO (Brookes et al., 2016) to deconvolute the contribution from oligomers. The curves obtained from dSuFu did not indicate higher order species and consequently did not need deconvolution. The subtracted frames, corresponding to the deconvoluted peak with a stable

$\mathrm{Rg}$, were selected to produce the protein scattering curve of scattered intensity as a function of the momentum transfer $\mathrm{q}=4 \pi \sin (\theta) / \lambda$, where $2 \theta$ is the diffusion angle and $\lambda$ is the wavelength. Further analysis (Guinier and Kratky plots, Radius of gyration Rg and intensity at origin $\mathrm{I}_{0}$ calculation) was performed with the ATSAS program suite (Franke et al., 2017).

Five $a b$ initio envelope calculations were carried out using the DENSS software (Grant, 2018) using the RAW interface (Hopkins et al., 2017). These were input into the DAMMAVER (Volkov and Svergun, 2003 ) suite to produce an averaged protein envelope.

\section{4) Molecular modelling of dSuFu.}

The dSuFu crystal structure 4KMA was modified into an open conformation using Rosetta, (Kaufmann et al., 2010) based on the hSuFu structure (pdb ID 4KM9), as a template for relative domain orientation. This model structure, and the crystal structure 4KMA for $\mathrm{dSuFu}$, were used as the initial and final frame, respectively, to guide a linear morphing obtained through the program Chimera (Pettersen et al., 2004). Each of the 20 morphing frames has been minimised without any constraint using the AMBER force field as implemented in Chimera (Pettersen et al., 2004). The 20 frames have then been analysed through the program Foxs (Schneidman-Duhovny et al., 2013) in order to calculate the corresponding scattering curve and its agreement with experimental data. The frame with the curve having the lowest chi value with respect to the experimental data was retained as the experimental model.

\section{5) Molecular modelling of hSuFu missing regions}

$\mathrm{hSuFu}$ residue numbering is according to Uniprot entry Q9VG38. Our SAXS data was measured on fulllength hSuFu. The crystal structure of $\mathrm{hSuFu}$ PDB code 4KM9 was used as a starting point. It shows two ordered domains, but the first 20 aminoacids and 76 residues in the C-terminal domain are 
disordered. AllosModFoxs program (Weinkam et al., 2012) was used to build missing residues and the structure was further refined using YASARA (Krieger and Vriend, 2014) to ensure a good geometry, and the chi ${ }^{2}$ of the calculated scattering curve with respect to the experimental curve was 12.2. The model was then submitted to Dadimodo server https://dadimodo.synchrotron-soleil.fr (Rudenko et al., 2019) for a flexible refinement against the SAXS data by introducing flexibility in the HDR1 and the HDR2. Five models were generated and ranked according to the chi value. The two ordered domains: residues 30-255 for the $\mathrm{N}$-terminal domain and residues 259-268 and 356-472 for the $\mathrm{C}$-terminal domain were kept rigid while residues 1-29, 256-258 and 269-355 were left flexible. Another test was performed with a closed model starting from structure 4KMD on which the HDR1 and HDR2 regions obtained from the Yasara-refined hSuFu structure were grafted.

\section{6) Molecular modelling of $\mathrm{zSuFu}$}

zSuFu was modelled by homology using the Phyre2 server (Kelley et al., 2015). The best-ranked model was based on hSuFu structure PDB code 4KMH. Then missing residues in the IDR2 loop (73 residues) were added with the AllosModFoxs program (Weinkam et al., 2012), yielding Chi2 values between 22 and 80 . The best model was processed with Dadimodo, with residues $18-255$ for the N-terminal and residues 259 - 268 and 356 - 472 for the C-terminal domain being kept rigid while residues 1-19, 256258 and 279-355 were left flexible. Five models were generated and ranked according to the chi value. 
SuFu disordered region is disclosed by solution studies

\section{Results}

\section{1) Synchrotron radiation circular dichroism}

(SRCD) shows that truncated $h \mathrm{huFu} \Delta 30$ is well structured, but less stable than $\mathrm{hSuFu}$.

We measured SRCD spectra between 10 and $90^{\circ} \mathrm{C}$ for $\mathrm{dSuFu}, \mathrm{hSuFu}, \mathrm{hSuFu} \Delta 30$ and $\mathrm{zSuFu}$ in two buffers: A) HEPES HCl 50mM pH 7.5, $\mathrm{NaCl} 200 \mathrm{mM}$, glycerol 10\%, Dithiothreitol 2mM or B) bis-tris $50 \mathrm{mM} \mathrm{pH} \mathrm{5.5,} \mathrm{NaCl} 200 \mathrm{mM}$, glycerol 10\%. All proteins showed spectra typical of structured proteins at low temperature, and the spectra became shallower when the temperature was increased, as exemplified in Figure 1A for hSuFu. The spectra cross at an isobestic point characteristic of a two-state transition. Table 1 shows the unfolding temperatures evaluated from the variation of CD at $208 \mathrm{~nm}$ as a function of temperature fitted to a sigmoid function with Kaleidagraph. For hSuFu and hSuFu $\Delta 30$ the Tm was measured at $\mathrm{pH} 7.5$ with DTT and at $\mathrm{pH} 5.5$ without DTT. Both forms of hSuFu showed an increase in their $\mathrm{Tm}$ in the first condition, but $\mathrm{hSuFu}$ is more stabilised by $\mathrm{pH} 7.5$ and DTT than $\mathrm{hSuFu} \Delta 30$. Figure 1B shows the evaluation of secondary structure content using the BestSel program (Micsonai et al., 2015) at 10 and $60^{\circ} \mathrm{C}$. At low temperature, our SuFu constructs contain between 40 and $50 \%$ of regular secondary structure and the other residues are in random coil. All studied SuFu constructs become richer in beta strand and lose helical structure when the temperature is raised from 10 to $60^{\circ} \mathrm{C}$. This trend is observed in other helix-containing proteins such as myoglobin (Fändrich et al., 2003).

\section{2) SEC-SAXS and molecular modelling show that $\mathrm{dSuFu}$ is an elongated monomer in solution}

\section{with a closed conformation.}

The SAXS measurements were conducted at the SWING beamline at the SOLEIL synchrotron, using a High Pressure Liquid Chromatography injection system (Thureau et al., submitted). In view of the higher stability at pH 7.5 with DTT detected with SRCD, we decided to perform our SAXS studies in those conditions. The sample migrated on the size exclusion column, and produced a peak with a homogeneous Radius of gyration ( $\mathrm{Rg}$ ) (Supplementary Figure S2) which allows us to say that the resulting SAXS curve (Figure 2A, green curve) corresponds to a monodispere particule. The estimation of the Rg using the Guinier approximation indicates an absence of aggregates and gives an $\operatorname{Rg}$ value of $27.2 \AA$. The Shannon sampling formalism indicates that the data is accurate at least up to a scattering vector of 0.4 $\AA^{-1}$. Table 2 gives a list of all measured values. The dimensionless Kratky plot (Figure 2B, grey curve) has a bell shape and does not rise at high angles, which denotes that $\mathrm{dSuFu}$ has a mostly compact structure with very little disorder. The distance distribution function, $\mathrm{P}(\mathrm{r})$, calculated using an indirect Fourier transform, yielded a well-behaved curve with a maximum particle dimension, $\mathrm{D}_{\max }$, of $90 \AA$ and 
a maximum of the curve at $\mathrm{r}=35 \AA$ (Figure $2 \mathrm{C}$, grey curve). The calculated mass of $51 \mathrm{kDa}$, obtained with Primus and Vc calculation (Rambo and Tainer, 2013), and the Rg values of $27.2 \AA$ and $27.3 \AA$ calculated from the Guinier and $\mathrm{P}(\mathrm{r})$ functions, respectively confirm that the particle is a monomer and is monodisperse in solution. The average envelope resulting from ab initio calculations encloses most of the crystal structure of $\mathrm{dSuFu} 4 \mathrm{KMA}$ with the exception of the 274-282 loop, which is mobile in the crystal structure, as shown by the thinner radius of the ribbon representation (Figure 3A). This figure highlights the IDR2 region that lines the C-terminal domain (residues 308-340 on the bottom left of the figure). It is ordered but it has no regular secondary structure.

The scattering curve calculated from the 4KMA crystal structure fits rather well with the SAXS spectrum, however the chi value is 7.3 and there is some disagreement between 0.15 and $0.25 \AA^{-1} \mathrm{q}$ value (Figure 3B, blue experimental curve, compare with 4KMA curve). We performed a modelling study in order to better characterise possible movements in $\mathrm{dSuFu}$ and find a model that fits the SAXS data better. The following two-step procedure was applied. First, we analysed the possible deformations in the $\mathrm{SuFu}$ structures. Wide rearrangements likely accounting for a conformational change in the dSuFu crystal structure have been inferred through hinge detection and compared with hSuFu crystal structures. For each structure, the Hingeprot program (Emekli et al., 2008) shows the two slowest modes of movement. For the 4KMA crystal structure, the slowest mode describes the bending of the two domains around residues Q252-D253 (Fig 3C). The second mode is described by three rigid blocks, namely N-terminal (M1 to C151 and Q177 to C194), central (Y152 to A176, and Q195 to A263) and C-terminal (G264 to E454), coloured in red, white and blue, respectively, in Figure 3D. The N- and C-terminal domains also describe a bending motion, but here the domains do not pivot on the same hinge because of the rigidity of the central block (see magenta arrows in Fig. 3D). Additionally, a wide fluctuation is observed in the loop A105-P128.

In the next step, we sampled the movements of the two domains along the direction of the bending mode in order to check if a better agreement with our SAXS results can be obtained by modelling an intermediate on this bending pathway. Two extreme open and closed conformations were generated for $\mathrm{dSuFu}$, using the open (4KM9) and closed (4KMD) conformations of hSuFu as templates. The conformational change from one state to the other was modelled using linear morphing, and twenty additional intermediate conformations were generated, each of which was regularized through energy minimization, (see materials and methods section). The SAXS profiles were then simulated for each of these energy-minimised conformations and compared to the experimental profile. Chi values calculated 
up to a q value of $0.4 \AA^{-1}$ are as follows: open model, 5.0; closed model, 7.7; 4KM9, 6.5; 4KMA, 7.3; best model, 3.8. The intermediate model whose diffusion curve was closest to the SAXS data was selected as the best solution (Figure 3B purple curve). This best model and the 4KMA structure of dSuFu have an RMSD of $0.7 \AA$ for all backbone atoms. The differences mostly concern the C-terminal domain whose RMSD, with respect to the crystallized counterpart, is $0.9 \AA$ versus $0.4 \AA$ for the N-terminal domain. Although the secondary structure is globally conserved between the proposed solution model and the crystal structure, the residues ranging from A294 - D332 in the latter are more closely packed in the domain core. The higher flexibility of these residues, containing the unresolved loop D296 - K308, accounts for a widening of the cleft between the two domains as shown by the minimum distance between the two domains ( $5 \AA$ between G133-Q302 C-alpha of 4KMA structure versus $11 \AA$ of the equivalent G147-R309 in the model). Finally, we used Dadimodo server to refine models for dSuFu starting from our best model and defined a flexible linker between the two domains consisting of residues 256-265, leaving the C-terminal domain free to move with respect to the $\mathrm{N}$-terminal domain. The five resulting structures are highly similar to each other and have an average $\mathrm{chi}^{2}$ of $1.81 \pm 0.04$ (Figure $4 \mathrm{~A}$ C and Table 3).

In summary, SAXS measurements show that the solution structure of dSuFu is slightly more open than the 4KMA dSuFu crystal structure, but is nevertheless different from the free human protein 4KM9. In addition, hinge detection shows that the clamp mode likely characterises SuFu motion. Finally, the region corresponding to IDR2 caps the C-terminal domain and is not disordered in the drosophila protein. Next, we decided to study the solution structure of vertebrate $\mathrm{SuFu}$.

\section{3) SEC-SAXS shows that the IDR2 disordered region spans a large volume for hSuFu and}

\section{zSuFu.}

We chose to compare proteins from vertebrates that are far from each other in evolution: zebra fish and human. hSuFu shows $36 \%$ identity with $\mathrm{dSuFu}$ and $81 \%$ identity with $\mathrm{zSuFu}$, while $\mathrm{zSuFu}$ has $35 \%$ identity with dSuFu (Fig S1). We measured SEC-SAXS data on purified hSuFu, hSuFu $\triangle 30$ and zSuFu. A reducing agent was necessary to remove a dimer-like species at $\mathrm{pH} 7.5$, coherent with the neutral $\mathrm{pKa}$ of cysteine protonation. We suspect that, at high $\mathrm{pH}$, a disulphide bridge is formed involving Cysteine 292 located in the IDR2 (data not shown).

The scattering curves are shown on Figure 2A and the Radii of gyration ( $\mathrm{Rg}$ ) values are listed in Table 2. The Shannon sampling formalism shows that all curves can be used at least up to $0.4 \AA^{-1}$ (Shannon 
and Weaver, 1949). Associated to the dimensionless Kratky curves shown on Figure 2B, our data show that $\mathrm{hSuFu}$ (dark blue curve), hSuFu $\Delta 30$ (black curve) and zSuFu (tuquoise curve) span a much larger volume than $\mathrm{dSuFu}$ (green curve). Indeed, for comparable molecular weights, the radius of gyration is $25 \%$ higher in $\mathrm{hSuFu}$ and $\mathrm{zSuFu}$ than for $\mathrm{dSuFu}$. This is in agreement with the presence of the IDR2 in zebrafish and human, which is ordered in drosophila. A comparison between the results of full-length $\mathrm{hSuFu}$ and truncated $\mathrm{hSuFu} \triangle 30$ indicates the contribution of the IDR2 to the total volume. The largest interatomic distance $\mathrm{D}_{\max }$ is $140 \AA$, only slightly decreased by the truncation, showing that the IDR2 contributes to the majority of the $\mathrm{D}_{\max }$ difference between drosophila $(90 \AA)$ and human $(150 \AA)$.

We went on to generate molecular models of $\mathrm{SuFu}$ whose calculated scattering curves fit the experimental curve well.

For full-length $\mathrm{hSuFu}$, as explained in the Methods section, we started from free hSuFu 4KM9 and built the missing regions using AllosModFoxS (Weinkam et al., 2012). We ran YASARA (Krieger and Vriend, 2014) to regularise the geometry and then used the Dadimodo software (Rudenko et al., 2019) to find models that fit the experimental curve better. In addition, as dSuFu domains are more closed even in the absence of the Gli peptide ligand, we wanted to check if $\mathrm{hSuFu}$ could harbor a closed conformation in solution. We thus generated a second starting model using the Gli peptide-bound conformation of $\mathrm{hSuFu} 4 \mathrm{KMD}$ on which the missing regions were attached similarly to the extended model.

For the elongated models, the average $\mathrm{chi}^{2}$ value is $1.73 \pm 0.01$ (Table 3). Figure $4 \mathrm{D}$ shows the superposition of the five models calculated. The N-terminal and C-terminal domains are in open conformation with respect to each other and the N-terminal disordered residues seem to have a rather compact structure. This is different from the IDR2 loops that protrude a long way into the solvent, conferring a very elongated structure to $\mathrm{hSuFu}$. A series of ab initio envelopes were calculated with the DENSS program and averaged with the DAMAVER suite. The elongated envelope is in agreement with the hypothesis that IDR2 spans a large volume in solution (figures 4E). However, we consider that the most significant validation is the fit between the model and the experimental scattering curves (figure 4F). In order to further assess the conformation of the IDR2 domains we ran Dadimodo starting from the second model that had a closed conformation of the two domains. The resulting models have an average $\mathrm{chi}^{2}$ of $2.80 \pm 0.3$. Their fit is significantly less good than those of the elongated models, showing that this set of conformations is less probable in solution. However, we cannot exclude that the protein spends part of its time in this closed conformation. 
In summary, $\mathrm{hSuFu}$ has a very elongated conformation in solution, compatible with an open conformation of the two ordered domains and an extended IDR.

We then compared our results with those obtained with N-terminal truncated hSuFu $\triangle 30$ using the elongated YASARA model as a starting point. Dadimodo provided five models with an average chi ${ }^{2}$ of $1.77 \pm 0.017$ (Table 3). As shown on Figure 4G, the models are slightly more variable than those of fulllength $\mathrm{hSuFu}$. The $\mathrm{C}$-terminal domain moves more with respect to the $\mathrm{N}$-terminal one and the IDR2 loop has different relative orientations. This observation may be due to a noisier scattering curve (Figures 2A and $4 \mathrm{H}$ ), but probably unrelated to its lower stability, as the Tm's obtained with SRCD (see above) are comparable. Finally, The DENSS envelope has a shape similar to that of full-length hSuFu (figure 4I), in agreement with a $9 \%$ lower Dmax value.

The zebrafish SAXS data confirm that the solution structure of zSuFu is very similar to that of human $\mathrm{SuFu}$, both in terms of dimensions (Table 2), models and ab initio envelope (Figure 4J-K), and significantly different from those of $\mathrm{dSuFu}$. The five Dadimodo refined models have $\mathrm{chi}^{2}$ values of $1.18 \pm 0.06$ (Figure 4L and Table 3) and have extended IDR2 loops similarly to hSuFu models.

In summary, our SAXS measurements provide structural data that the crystal structures do not supply. They show that drosophila $\mathrm{SuFu}$ has a very different shape in solution than vertebrate $\mathrm{SuFu}$. This difference is mainly due to the presence of an extended IDR2 similar in both human and zebrafish species.

\section{Discussion and conclusion}

Our solution investigation disclosed differences between drosophila and vertebrate $\mathrm{SuFu}$ that could not be anticipated from their respective crystal structures. Our SAXS results show that the closed conformation of $\mathrm{dSuFu}$ is not due to crystal packing effects but is intrinsic to $\mathrm{dSuFu}$, and our modelling results indicate that this may be due to different hierarchy in the hinge motions, leading to a different equilibrium position. In addition, the sequence corresponding to the Intrinsically Disordered Region IDR2 is ordered in drosophila, thus stabilising the two domains in this closed conformation.

The hSuFu protein has an elongated monomeric structure and SAXS data allowed us to build models for the missing residues at the $\mathrm{N}$-terminal and in the $\mathrm{C}$-terminal domain. Those models suggest that the IDR2 forms an elongated loop that protrudes into the solvent. This is comforted by the study of a 
truncated mutant $\mathrm{hSuFu} \triangle 30$ and of $\mathrm{zSuFu}$, which is $81 \%$ identical in amino acid sequence to $\mathrm{hSuFu}$ and whose SAXS-derived size and shape is very similar to that of $\mathrm{hSuFu}$.

The IDR2 loop thus contributes strongly to the large values of $\mathrm{Rg}$ and $\mathrm{D}_{\max }$ of those proteins, and we investigated its amino acid composition in order to predict how the IDR2 of other species might behave. The IDR2 is 76 and 73 residue-long in human and zebrafish, respectively.

In the sequences aligned in Figure S1, the IDR2 starts with an aromatic residue W/F (W275 in human) belonging to the first strand of the first $\beta$-sheet of the C-terminal domain. This aromatic establishes contacts that stabilise the interaction with conserved hydrophobic residues in the second $B$-sheet. The last residues in the IDR2 are the first ones of this $\beta$-sheet and they ensure the stabilisation of the loop ends. Disorder prediction using the Prediction of Natural Disorder PONDR server (http://www.pondr.com) shows that all sequences have a disorder tendency. Figure 5A shows the PONDR plot for the three proteins studied in this work. It confirms that $\mathrm{dSuFu}$ has a shorter disordered segment than zSuFu and hSuFu. In fact, if we consider the recommended disorder index threshold of 0.5 , the PONDR metaserver predicts a stretch of 60 disordered residues while the crystal structure of dSuFu 4KMA shows only 11 disordered residues, confirmed by our SAXS data. Only when raising the threshold to 0.9 does the PONDR result almost predict the regions disordered in the crystal. On the other hand, with a threshold of 0.6, PONDR predicts a 90-residue-long disordered stretch for the human and zebrafish sequences, which is very close to the missing region in the crystal structures of $\mathrm{hSuFu}$. This is in agreement with the observation that drosophila IDR2 region is much less disordered than the vertebrate one.

The elongation of IDR2 loop in vertebrates might be associated with an increased complexity in the Hh pathway from flies to vertebrates. This is exemplified by the transcription factor Cubitus interruptus (Ci) that is unique in drosophila, while three Gli proteins are simultaneously present in mammals: Gli1, Gli2 and Gli3 (Ruppert et al., 1988), allowing fine-tuning of the gene expression pattern via other partner proteins.

The function of the IDR2 region is not well known. However, Cherry et al. have studied the effect of its removal on the Hedgehog pathway activation. They found that the IDR2 is dispensable for Gli binding and Hh pathway activation with a luciferase reporter in HEK cells. However, activation of the Hh pathway in mouse embryonic fibroblast cells was repressed in the presence of full-length hSuFu but not in the presence of IDR2-truncated hSuFu. Moreover, a chimeric human protein harbouring drosophila IDR2 failed to repress the pathway (Cherry et al., 2013). This confirms that IDR2 of vertebrate SuFu 
has a role in the regulation of the Hh pathway, likely linked to post-translation modifications and nuclear trafficking. The amino acid composition of SuFu sequences as displayed in Table 4 shows that the IDR2, as compared to the overall sequence, is richer in acidic residues and, to a lesser extent, in basic and polar residues, and poorer in hydrophobic residues, as could be expected from the nature of IDRs. The "Phosphosite" data base (Hornbeck et al., 2004) indicates that ten out of sixteen post-transcriptional modifications of $\mathrm{hSuFu}$ occur in the 80 aminoacids of the IDR2. Six serine and threonine residues are predicted to be phophorylated and three lysines are predicted to be ubiquitylated or acetylated. Moreover, Zhang et al. showed that the mammalian SuFu harbours a nuclear export signal spanning residues 308318 and two phosphorylatable serines 342 and 346 (Zhang et al., 2017). As shown on Figure 5B, those residues are located in the IRD2 making them very accessible to interact with kinases and the nuclear pore complex. This property makes the IRD2 a very good candidate to interact with exportins similarly to the complex between exportin CRM1, Ran-GTP and import adapter snurportin1 (SPN1), that involves disordered regions of SPN1 in contact with CRM1 (Monecke et al., 2009). It is known that SuFu interacts with $\mathrm{Ci} / \mathrm{Gli}$ and several other partners such as SAP18, galectin3 or p66ß (Lin et al., 2014; Paces-Fessy et al., 2004). The difference between vertebrate and drosophila $\mathrm{SuFu}$ is further underlined by the observation that phosphorylated serines in $\mathrm{dSuFu}$ do not seem necessary for Hh pathway repression (Oh et al., 2015).

In conclusion, our biophysical studies show that the disordered region of Suppressor of Fused has different lengths and properties in insects and vertebrates. In vertebrates, it is highly dynamic and spans large areas, allowing it to interact with post-translational modifying enzymes. Looking for partners specifically able to bind the IDR2 would help understand the role of this peculiar appendix.

\section{Acknowledgements}

We are very grateful to Marie-Hélène Le Du, Dominique Durand and Patrice Vachette (Institut de Biologie Intégrative de la Cellule, CEA, CNRS, Université Paris-Saclay, France.) for help with data processing software, the SOLEIL synchrotron for access to beamlines, Javier Perez at Swing beamline, and Frank Wien and Mathieu Refrigiers at Disco beamline. Molecular graphics figures and analyses were performed with UCSF Chimera, developed by the Resource for Biocomputing, Visualization, and Informatics at the University of California, San Francisco, with support from NIH P41-GM103311.

\section{Funding}

AJ was financed by a thesis grant from Ministère de l'Enseignement Supérieur et de la Recherche and 
by a fourth year thesis grant from Association pour la Recherche contre le Cancer (ARC). SM was financed by a thesis grant from Ministère de l'Enseignement Supérieur et de la Recherche - IDEX Paris Sciences Lettres. This work was supported by the Centre National de la Recherche Scientifique, and by the "Initiative d'Excellence" program from the French State (Grant "DYNAMO", ANR-11-LABEX0011-01) and Fondation ARC pour la recherche sur le cancer (grant 1112) to APl and MS.

\section{Author contribution}

$\mathrm{SM}, \mathrm{AJ}$ and APa cloned and purified proteins. SM, AJ and VB measured and processed CD and SAXS data. VB, MB and FO performed molecular modelling and analysis. AT and OR helped with Dadimodo modeling and data processing. APl and MS provided constructs and background about Hh pathway. VB designed the research, conducted research and wrote the manuscript with the help of all authors.

\section{Abbreviations}

$\mathrm{Ci}$, cubitus interruptus

Hh, Hedgehog

IDR, intrinsically disordered region

Ptc, Patched

SAXS, small angle X-ray scattering

Smo, Smoothened

SRCD, synchrotron radiation circular dichroism

$\mathrm{SuFu}$, Suppressor of Fused 


\section{Tables}

Table 1

$\begin{array}{lll}\text { Protein } & \text { Buffer } & \text { Tm } \\ \text { zSuFu } & \text { HEPES pH 7.5, NaCl 200, glycerol 10\%, DTT 2mM } & 49.9^{\circ} \\ \text { hSuFu } & \text { HEPES pH 7.5, NaCl 200, glycerol 10\%, DTT 2mM } & 60.0^{\circ} \\ \text { hSuFu } & \text { bis-tris pH 5.5 50mM, NaCl 200, glycerol 10\% } & 39.1^{\circ} \\ \text { hSuFu } \Delta 30 & \text { HEPES pH 7.5, NaCl 200, glycerol 10\%, DTT 2mM } & 53.6^{\circ} \\ \text { hSuFu } \Delta 30 & \text { bis-tris pH 5.5 50mM, NaCl 200, glycerol 10\% } & 38.8^{\circ}\end{array}$

Table 1: transition temperature for studied proteins measured by SRCD 


\section{Table 2}

Sample

Data collection parameters

Beamline

Energy (keV)

Sample-detector distance 12

1804 (mm)

q-range $(\AA ̊-1)$

Exposure time

detector

$0.008-0.5$

1s

Aviex charge coupled

device

software

Primary data reduction

Data processing

Ab initio analysis

Computation of model

intensities

Model building

Model refinement

27.2

$\mathrm{qRg}_{\max }$

Dmax frm $P(r)(\AA ̊)$

90

Rg from $P(r)(\AA)$

27.3

$\mathrm{I}_{0}$ from $\mathrm{P}(\mathrm{r})(\mathrm{cm}-1)$

1.747

$\mathrm{Ch}^{\mathrm{i} 2} \mathrm{Gnom}$

Dmax/Rg

$\mathrm{qRg}_{\max }$ (Kratky)

1.6

$\mathrm{q}_{\text {opt }}\left(\AA^{-1}\right)$ (Shannon)
Dadimodo

0.017

1.27

2.0

3.3

Foxtrot

US-SOMO

GNOM V5.0, DENSS

Crysol

Modeller

$4 \mathrm{km9+}$

AllosModFoxS

Dadimodo

31.5

36.0

0.03

1.2

150

38.1

0.03

1.56

4.2

2.0

0.49
12

2000

$0.008-0.5$

0.008-0.5

990ms
12

2000

Eiger 4M
zSuFu

Table 2: SAXS data for dSuFu, hSuFu and zSuFu

$\mathrm{Rg}=$ radius of gyration; Dmax= maximum interatomic distance; $q$ Rgmax= abscises of the maximum value for the normalised Kratky plot shown in Figure 3B.

Page 18 


\section{Table 3}

\begin{tabular}{|l|l|l|l|l|}
\hline sample & dSuFu & hSuFu $\Delta 30$ & hSuFu & zSuFu \\
\hline $\begin{array}{l}\text { CRYSOL on Dadimodo output (5 } \\
\text { structures) }\end{array}$ & & & & \\
\hline q range $\left(\AA^{-1}\right)$ & $0.008-0.4$ & $0.008-0.4$ & $0.008-0.4$ & $0.008-0.4$ \\
\hline Chi $^{2}$ & $1.80 \pm 0.04$ & $1.77 \pm 0.02$ & $1.73 \pm 0.01$ & $1.18 \pm 0.06$ \\
\hline Hydration shell contrast Dro $\left(e-\AA^{3}\right)$ & 0.030 & 0.045 & 0.03 & 0.03 \\
\hline Ra $\AA$ & 1.617 & 1.614 & 1.615 & 1.615 \\
\hline
\end{tabular}

Table 3: atomistic modelling of structures from SAXS data 


\section{Table 4}

\begin{tabular}{|c|c|c|c|}
\hline aminoacid & $\%$ overall & \% IDR2 & \%IDR2-\%overall \\
\hline$A$ & 5.38 & 5.12 & -0.26 \\
\hline C & 1.61 & 2.33 & 0.72 \\
\hline$D$ & 6.50 & 8.84 & 2.34 \\
\hline$E$ & 7.62 & 9.30 & 1.69 \\
\hline $\mathrm{F}$ & 3.84 & 1.40 & -2.45 \\
\hline G & 7.55 & 3.26 & -4.29 \\
\hline $\mathrm{H}$ & 3.14 & 1.86 & -1.28 \\
\hline I & 4.82 & 6.51 & 1.69 \\
\hline K & 3.91 & 6.05 & 2.13 \\
\hline L & 10.76 & 7.44 & -3.32 \\
\hline M & 2.10 & 1.40 & -0.70 \\
\hline $\mathrm{N}$ & 3.00 & 3.72 & 0.72 \\
\hline$P$ & 8.04 & 8.37 & 0.34 \\
\hline$Q$ & 5.17 & 6.51 & 1.34 \\
\hline $\mathrm{R}$ & 5.31 & 8.84 & 3.53 \\
\hline$S$ & 6.92 & 13.95 & 7.04 \\
\hline $\mathrm{T}$ & 5.24 & 3.72 & -1.52 \\
\hline V & 5.10 & 1.40 & -3.71 \\
\hline W & 1.82 & 0.00 & -1.82 \\
\hline Y & 2.17 & 0.00 & -2.17 \\
\hline
\end{tabular}

Table 4: amino acid composition of the three SuFu sequences displayed in Figure S1. The percentages are calculated using the Protparam server (https://web.expasy.org). The first column corresponds to all amino acids of the sequences; the second column corresponds to the IDR2 aminoacids as shown in the dotted black box on Figure S1. The third column is the difference between the IDR2 and the overall composition. 


\section{Figure legends}

Figure 1: SRCD measurements of $\mathrm{SuFu}$ from different species. A, CD spectra showing thermal unfolding of $\mathrm{hSuFu}$ at $\mathrm{pH}$ 7.5. B, secondary structure evaluation from SRCD spectra for all studied proteins at 10 and $60^{\circ} \mathrm{C}$.

Figure 2: SAXS studies of hSuFu WT and $\Delta 30, \mathrm{dSuFu}$ and zSuFu. Green, dSuFu; dark blue, hSuFu WT; black, hSuFu $\triangle 30$; light blue, zSuFu. A, SAXS scattering curves B, dimensionless Kratky plots; $\mathrm{C}$, pair distance distribution $\mathrm{P}(\mathrm{R})$.

Figure 3: SAXS structure of drosophila SuFu. A, superposition of the crystal structure 4KMA and the envelope calculated by GASBOR and DAMAVER suite. The structure is displayed using a worm-like representation with a radius inversely proportional to the B-factor in the PDB file. B, scattering curves superposed using Crysol (top) and their differences (bottom). The experimental curve is in light blue, the PDB models 4KM9 (hSuFu) in red, 4KMA (dSuFu) in mustard green, the three models used in the normal mode simulation: closed in green, open in orange and best model in purple. C, D, the two slowest modes of movement calculated by Hingeprot. The colours distinguish the domains moving relative to each other and the arrows show the direction of movements.

Figure 4: Modelling of dSuFu (A-C) hSuFu WT (D-F), hSuFu $\triangle 30$ (G-I), zSuFu (J-L) and from SAXS data. A, D, G, J: Five best models from Dadimodo; B E, I, K, rotated models superimposed with envelope from DENSS; C, F, H, L, fitting of experimental and model scattering curves.

Figure 5: Intrinsically disordered region of $\mathrm{SuFu}$ : disorder prediction and structural features. A, PondR prediction of protein disorder for the three sequences studied in this work; $\mathrm{B}, \mathrm{hSuFu}$ model obtained from our SAXS data showing phosphorylatable serines 290, 301, 342, 346, 349, 352 and threonines 305 and 353 are coloured black; ubiquitylated lysines 303, 321 and 344 are colored orange (Akimov et al., 2018) and nuclear export sequence (residues 308-318) is coloured blue (Zhang et al, 2017). 


\section{Supplementary figures legends}

Figure S1: alignment of SuFu aminoacid sequences from human, danio rerio and drosophila melanogaster, showing aminoacid numbering on the sides. Top line $=$ conservation histogram; dotted grey box= IDR1; dotted black box=IDR2 for human and zebrafish; dotted red box = IDR2 for drosophila; N-terminal domain is in light blue boxes and C-terminal domain is in pink boxes. the wavy blue box shows the nuclear export signal and arrows show phosphorylable serines in the vertebrate IDR2. Green background= alpha helix; yellow background $=$ beta strand

Figure $\mathrm{S} 2: \mathrm{dSuFu}, \mathrm{I}_{0}$ and $\mathrm{Rg} \mathrm{SAXS}$ profile as a function of image index.

Figure S3: hSuFu I0, Rg SAXS profile as a function of image index after baseline subtraction with USSOMO.

Figure S4: zSuFu I, Rg SAXS profile as a function of image index after baseline subtraction with USSOMO. 


\section{References}

Akimov, V., Barrio-Hernandez, I., Hansen, S.V.F., Hallenborg, P., Pedersen, A.-K., Bekker-Jensen, D.B., Puglia, M., Christensen, S.D.K., Vanselow, J.T., Nielsen, M.M., Kratchmarova, I., Kelstrup, C.D., Olsen, J.V., Blagoev, B., 2018. UbiSite approach for comprehensive mapping of lysine and Nterminal ubiquitination sites. Nat. Struct. Mol. Biol. 25, 631-640. https://doi.org/10.1038/s41594-0180084-y

Brookes, E., Vachette, P., Rocco, M., Pérez, J., 2016. US-SOMO HPLC-SAXS module: dealing with capillary fouling and extraction of pure component patterns from poorly resolved SEC-SAXS data. J. Appl. Crystallogr. 49, 1827-1841. https://doi.org/10.1107/S1600576716011201

Chen, Y., Yue, S., Xie, L., Pu, X.H., Jin, T., Cheng, S.Y., 2011. Dual phosphorylation of Suppressor of fused by PKA and GSK3beta regulates its stability and localization in the primary cilium. J Biol Chem 286, 13502-13511. https://doi.org/10.1074/jbc.M110.217604

Cherry, A.L., Finta, C., Karlstrom, M., Jin, Q., Schwend, T., Astorga-Wells, J., Zubarev, R.A., Del Campo, M., Criswell, A.R., de Sanctis, D., Jovine, L., Toftgard, R., 2013. Structural basis of SUFUGLI interaction in human Hedgehog signalling regulation. Acta Crystallogr. Sect. D 69, 2563-2579. https://doi.org/10.1107/S0907444913028473

Cooper, A.F., Yu, K.P., Brueckner, M., Brailey, L.L., Johnson, L., McGrath, J.M., Bale, A.E., 2005. Cardiac and CNS defects in a mouse with targeted disruption of suppressor of fused.

Development 132, 4407-4417.

Emekli, U., Schneidman-Duhovny, D., Wolfson, H.J., Nussinov, R., Haliloglu, T., 2008. HingeProt: automated prediction of hinges in protein structures. Proteins 70, 1219-27.

Fändrich, M., Forge, V., Buder, K., Kittler, M., Dobson, C.M., Diekmann, S., 2003. Myoglobin forms amyloid fibrils by association of unfolded polypeptide segments. Proc. Natl. Acad. Sci. 100, $15463-15468$.

Franke, D., Petoukhov, M.V., Konarev, P.V., Panjkovich, A., Tuukkanen, A., Mertens, H.D.T., Kikhney, A.G., Hajizadeh, N.R., Franklin, J.M., Jeffries, C.M., Svergun, D.I., 2017. ATSAS 2.8: a comprehensive data analysis suite for small-angle scattering from macromolecular solutions. J. Appl. Crystallogr. 50, 1212-1225. https://doi.org/10.1107/S1600576717007786

Grant, T.D., 2018. Ab initio electron density determination directly from solution scattering data. Nat. Methods 15, 191-193. https://doi.org/10.1038/nmeth.4581

Hooper, J.E., Scott, M.P., 1989. The Drosophila patched gene encodes a putative membrane protein Page 23 
required for segmental patterning. Cell 59, 751-765.

Hopkins, J.B., Gillilan, R.E., Skou, S., 2017. BioXTAS RAW: improvements to a free open-source program for small-angle X-ray scattering data reduction and analysis. J. Appl. Crystallogr. 50, 15451553. https://doi.org/10.1107/S1600576717011438

Hornbeck, P.V., Chabra, I., Kornhauser, J.M., Skrzypek, E., Zhang, B., 2004. PhosphoSite: A bioinformatics resource dedicated to physiological protein phosphorylation. PROTEOMICS 4, 15511561. https://doi.org/10.1002/pmic.200300772

Hu, A., Song, B.-L., 2019. The interplay of Patched, Smoothened and cholesterol in Hedgehog signaling. Curr. Opin. Cell Biol. 61, 31-38. https://doi.org/10.1016/j.ceb.2019.06.008

Huangfu, D., Liu, A., Rakeman, A.S., Murcia, N.S., Niswander, L., Anderson, K.V., 2003.

Hedgehog signalling in the mouse requires intraflagellar transport proteins. Nature 426, 83-87. https://doi.org/10.1038/nature02061

Ingham, P.W., Taylor, A.M., Nakano, Y., 1991. Role of the Drosophila patched gene in positional signalling. Nature 353, 184. https://doi.org/10.1038/353184a0

Jabrani, A., Makamte, S., Moreau, E., Gharbi, Y., Plessis, A., Bruzzone, L., Sanial, M., Biou, V., 2017. Biophysical characterisation of the novel zinc binding property in Suppressor of Fused. Sci. Rep. 7, 11139. https://doi.org/10.1038/s41598-017-11203-2

Jiang, J., Hui, C., 2008. Hedgehog Signaling in Development and Cancer. Dev. Cell 15, 801-812. https://doi.org/10.1016/j.devcel.2008.11.010

Kaufmann, K.W., Lemmon, G.H., DeLuca, S.L., Sheehan, J.H., Meiler, J., 2010. Practically Useful: What the Rosetta Protein Modeling Suite Can Do for You. Biochemistry 49, 2987-2998. https://doi.org/10.1021/bi902153g

Kelley, L.A., Mezulis, S., Yates, C.M., Wass, M.N., Sternberg, M.J.E., 2015. The Phyre2 web portal for protein modeling, prediction and analysis. Nat. Protoc. 10, 845-858.

https://doi.org/10.1038/nprot.2015.053

Koudijs, M.J., den Broeder, M.J., Keijser, A., Wienholds, E., Houwing, S., van Rooijen, E.M.H.C., Geisler, R., van Eeden, F.J.M., 2005. The Zebrafish Mutants dre, uki, and lep Encode Negative Regulators of the Hedgehog Signaling Pathway. PLoS Genet. 1, e19.

https://doi.org/10.1371/journal.pgen.0010019

Krieger, E., Vriend, G., 2014. YASARA View—molecular graphics for all devices-from smartphones to workstations. Bioinformatics 30, 2981-2982.

Page 24 
https://doi.org/10.1093/bioinformatics/btu426

Lees, J.G., Smith, B.R., Wien, F., Miles, A.J., Wallace, B.A., 2004. CDtool—an integrated software package for circular dichroism spectroscopic data processing, analysis, and archiving. Anal. Biochem. 332, 285-289. https://doi.org/10.1016/j.ab.2004.06.002

Lin, C., Yao, E., Wang, K., Nozawa, Y., Shimizu, H., Johnson, J.R., Chen, J.-N., Krogan, N.J., Chuang, P.-T., 2014. Regulation of Sufu activity by p66ß and Mycbp provides new insight into vertebrate Hedgehog signaling. Genes Dev. 28, 2547-2563. https://doi.org/10.1101/gad.249425.114

Marigo, V., Davey, R.A., Zuo, Y., Cunningham, J.M., Tabin, C.J., 1996. Biochemical evidence that Patched is the Hedgehog receptor. Nature 384, 176-179.

Maurya, A.K., Ben, J., Zhao, Z., Lee, R.T.H., Niah, W., Ng, A.S.M., Iyu, A., Yu, W., Elworthy, S., van Eeden, F.J.M., Ingham, P.W., 2013. Positive and Negative Regulation of Gli Activity by Kif7 in the Zebrafish Embryo. PLoS Genet. 9, e1003955. https://doi.org/10.1371/journal.pgen.1003955

Micsonai, A., Wien, F., Kernya, L., Lee, Y.-H., Goto, Y., Réfrégiers, M., Kardos, J., 2015. Accurate secondary structure prediction and fold recognition for circular dichroism spectroscopy. Proc. Natl. Acad. Sci. 112, E3095-E3103. https://doi.org/10.1073/pnas. 1500851112

Miroux, B., Walker, J.E., 1996. Over-production of proteins in Escherichia coli: mutant hosts that allow synthesis of some membrane proteins and globular proteins at high levels. J Mol Biol 260, 28998. https://doi.org/10.1006/jmbi.1996.0399

Monecke, T., Güttler, T., Neumann, P., Dickmanns, A., Görlich, D., Ficner, R., 2009. Crystal Structure of the Nuclear Export Receptor CRM1 in Complex with Snurportin1 and RanGTP. Science 324, 1087-1091. https://doi.org/10.1126/science.1173388

Monnier, V., Dussillol, F., Alves, G., Lamour-Isnard, C., Plessis, A., 1998. Suppressor of fused links Fused and Cubitus interruptus on the Hedgehog signalling pathway. Curr. Biol. 8, 583-586.

Nusslein-Volhard, C., Wieschaus, E., 1980. Mutations affecting segment number and polarity in Drosophila. Nature 287, 795-801.

Oh, S., Kato, M., Zhang, C., Guo, Y., Beachy, P.A., 2015. A Comparison of Ci/Gli Activity as Regulated by Sufu in Drosophila and Mammalian Hedgehog Response. PLOS ONE 10, e0135804. https://doi.org/10.1371/journal.pone.0135804

Paces-Fessy, M., Boucher, D., Petit, E., Paute-Briand, S., Blanchet-Tournier, M.-F., 2004. The negative regulator of Gli, Suppressor of fused (Sufu), interacts with SAP18, Galectin3 and other nuclear proteins. Biochem J 378, 353-362. https://doi.org/10.1042/bj20030786

Page 25 
Pak, E., Segal, R.A., 2016. Hedgehog Signal Transduction: Key Players, Oncogenic Drivers, and Cancer Therapy. Dev. Cell 38, 333-344. https://doi.org/10.1016/j.devcel.2016.07.026

Pettersen, E.F., Goddard, T.D., Huang, C.C., Couch, G.S., Greenblatt, D.M., Meng, E.C., Ferrin, T.E., 2004. UCSF Chimera--a visualization system for exploratory research and analysis. J Comput Chem 25, 1605-12. https://doi.org/10.1002/jcc.20084 [doi]

Pospisilik, J.A., Schramek, D., Schnidar, H., Cronin, S.J.F., Nehme, N.T., Zhang, X., Knauf, C., Cani, P.D., Aumayr, K., Todoric, J., Bayer, M., Haschemi, A., Puviindran, V., Tar, K., Orthofer, M., Neely, G.G., Dietzl, G., Manoukian, A., Funovics, M., Prager, G., Wagner, O., Ferrandon, D., Aberger, F., Hui, C., Esterbauer, H., Penninger, J.M., 2010. Drosophila Genome-wide Obesity Screen Reveals Hedgehog as a Determinant of Brown versus White Adipose Cell Fate. Cell 140, 148-160. https://doi.org/10.1016/j.cell.2009.12.027

Preat, T., 1992. Characterization of Suppressor of fused, a Complete Suppressor of the fused Segment Polarity Gene of Drosophila melanogaster. Genetics 132, 725-736.

Raducu, M., Fung, E., Serres, S., Infante, P., Barberis, A., Fischer, R., Bristow, C., Thézénas, M.L., Finta, C., Christianson, J.C., Buffa, F.M., Kessler, B.M., Sibson, N.R., Di Marcotullio, L., Toftgård, R., D’Angiolella, V., 2016. SCF (Fbx117) ubiquitylation of Sufu regulates Hedgehog signaling and medulloblastoma development. EMBO J. 35, 1400-1416.

https://doi.org/10.15252/embj.201593374

Rambo, R.P., Tainer, J.A., 2013. Accurate assessment of mass, models and resolution by smallangle scattering. Nature 496, 477-481. https://doi.org/10.1038/nature12070

Rudenko, O., Thureau, A., Perez, J., 2019. Evolutionary refinement of the 3D structure of multidomain protein complexes from small angle X-ray scattering data., in: Proceedings of the Genetic and Evolutionary Computation Conference Companion (GECCO '19). Presented at the GECCO '19, Manuel López-Ibáñez (Ed.), Prague, Czech Republic, pp. 401-402.

https://doi.org/10.1145/3319619.3322002

Ruppert, J.M., Kinzler, K.W., Wong, A.J., Bigner, S.H., Kao, F.T., Law, M.L., Seuanez, H.N., O’Brien, S.J., Vogelstein, B., 1988. The GLI-Kruppel family of human genes. Mol. Cell. Biol. 8, 3104-3113. https://doi.org/10.1128/MCB.8.8.3104

Schneidman-Duhovny, D., Hammel, M., Tainer, J., Sali, A., 2013. Accurate SAXS Profile Computation and its Assessment by Contrast Variation Experiments. Biophys. J. 105, 962-974. https://doi.org/10.1016/j.bpj.2013.07.020

Page 26 
Shannon, C., E., Weaver, warren, 1949. the mathematical theory of communication, University of Illinois. ed. Urbana.

Sisson, B.E., Ziegenhorn, S.L., Holmgren, R.A., 2006. Regulation of Ci and Su(fu) nuclear import in Drosophila. Dev. Biol. 294, 258-270.

Svard, J., Henricson, K.H., Persson-Lek, M., Rozell, B., Lauth, M., Bergström, A., Ericson, J., Toftgård, Rune, R., Teglund, S., 2006. Genetic Elimination of Suppressor of Fused Reveals an Essential Repressor Function in the Mammalian Hedgehog Signaling Pathway. Dev. Cell 10, 187-197.

Volkov, V.V., Svergun, D.I., 2003. Uniqueness of ab initio shape determination in small-angle scattering. J. Appl. Crystallogr. 36, 860-864. https://doi.org/10.1107/S0021889803000268

Wang, Y., Li, Y., Hu, G., Huang, X., Rao, H., Xiong, X., Luo, Z., Lu, Q., Luo, S., 2016. Nek2A phosphorylates and stabilizes $\mathrm{SuFu}$ : A new strategy of Gli2/Hedgehog signaling regulatory mechanism. Cell. Signal. 28, 1304-1313. https://doi.org/10.1016/j.cellsig.2016.06.010

Weinkam, P., Pons, J., Sali, A., 2012. Structure-based model of allostery predicts coupling between distant sites. Proc. Natl. Acad. Sci. 109, 4875-4880. https://doi.org/10.1073/pnas.1116274109

Yabut, O.R., Fernandez, G., Huynh, T., Yoon, K., Pleasure, S.J., 2015. Suppressor of Fused Is Critical for Maintenance of Neuronal Progenitor Identity during Corticogenesis. Cell Rep. 12, 20212034. https://doi.org/10.1016/j.celrep.2015.08.031

Zhang, D., Iyer, L.M., Aravind, L., 2011. A novel immunity system for bacterial nucleic acid degrading toxins and its recruitment in various eukaryotic and DNA viral systems. Nucleic Acids Res. $39,4532-4552$.

Zhang, Y., Bulkley, D.P., Xin, Y., Roberts, K.J., Asarnow, D.E., Sharma, A., Myers, B.R., Cho, W., Cheng, Y., Beachy, P.A., 2018. Structural Basis for Cholesterol Transport-like Activity of the Hedgehog Receptor Patched. Cell 175, 1352-1364.e14. https://doi.org/10.1016/j.cell.2018.10.026

Zhang, Y., Fu, L., Qi, X., Zhang, Z., Xia, Y., Jia, J., Jiang, J., Zhao, Y., Wu, G., 2013. Structural insight into the mutual recognition and regulation between Suppressor of Fused and Gli/Ci. Nat. Commun. 4, 1-12. https://doi.org/10.1038/ncomms3608

Zhang, Ziyu, Shen, L., Law, K., Zhang, Zengdi, Liu, X., Hua, H., Li, S., Huang, H., Yue, S., Hui, C.-C., Cheng, S.Y., 2017. Suppressor of Fused chaperones Gli proteins to generate transcriptional responses to Sonic Hedgehog signaling. Mol. Cell. Biol. 37, e421.

https://doi.org/10.1128/MCB.00421-16 
A

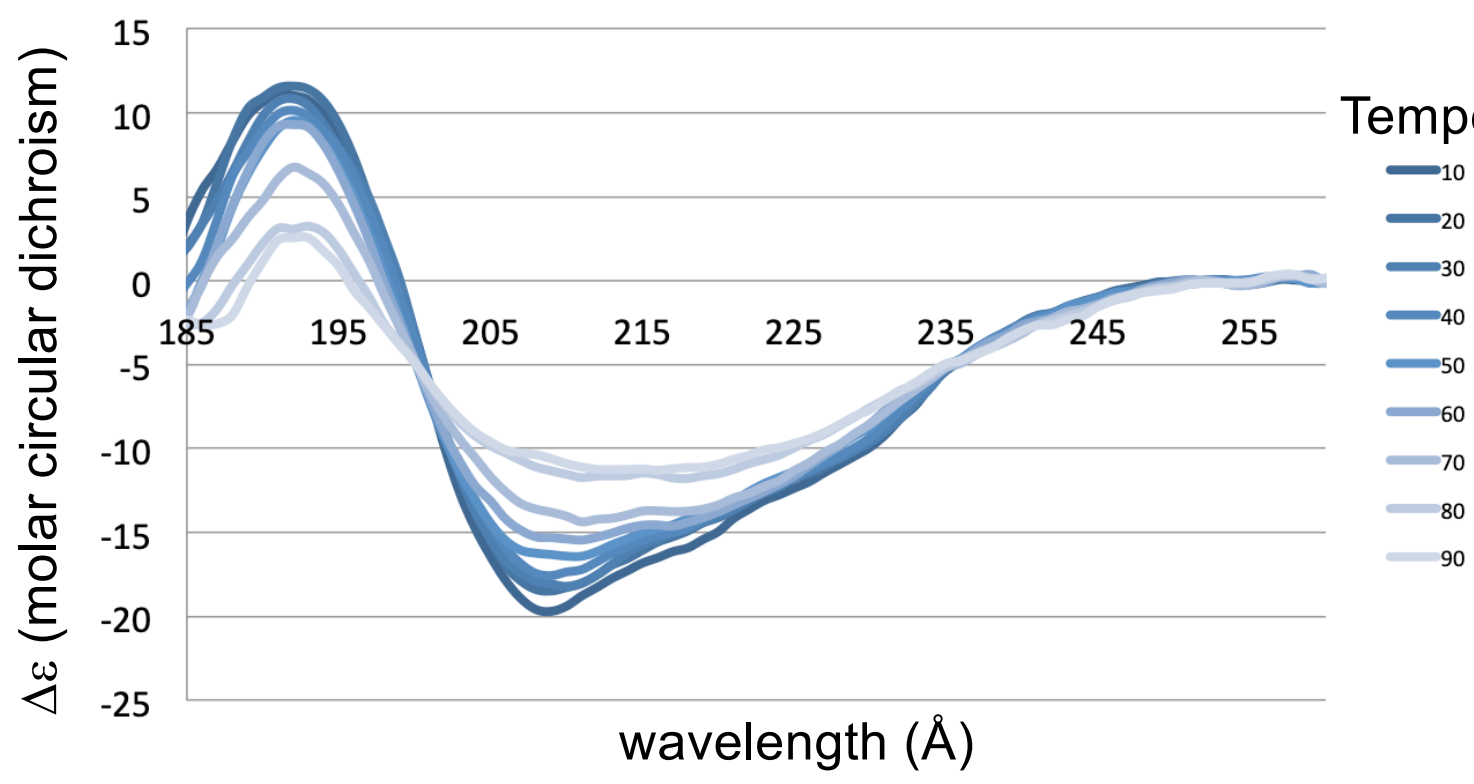

Figure 1

B

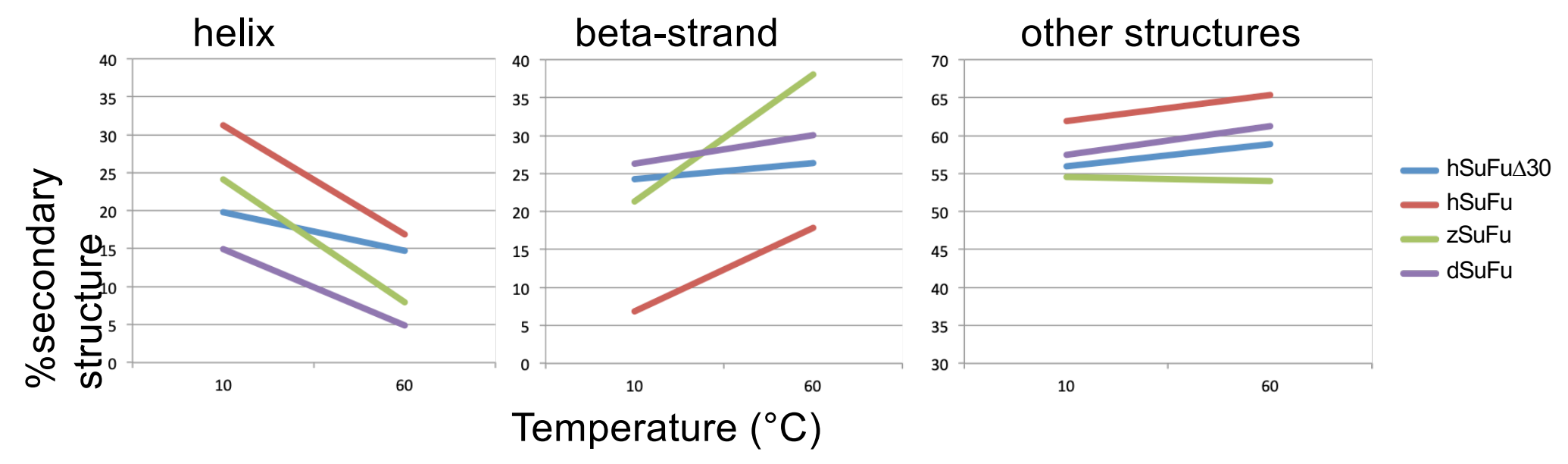


Figure 2
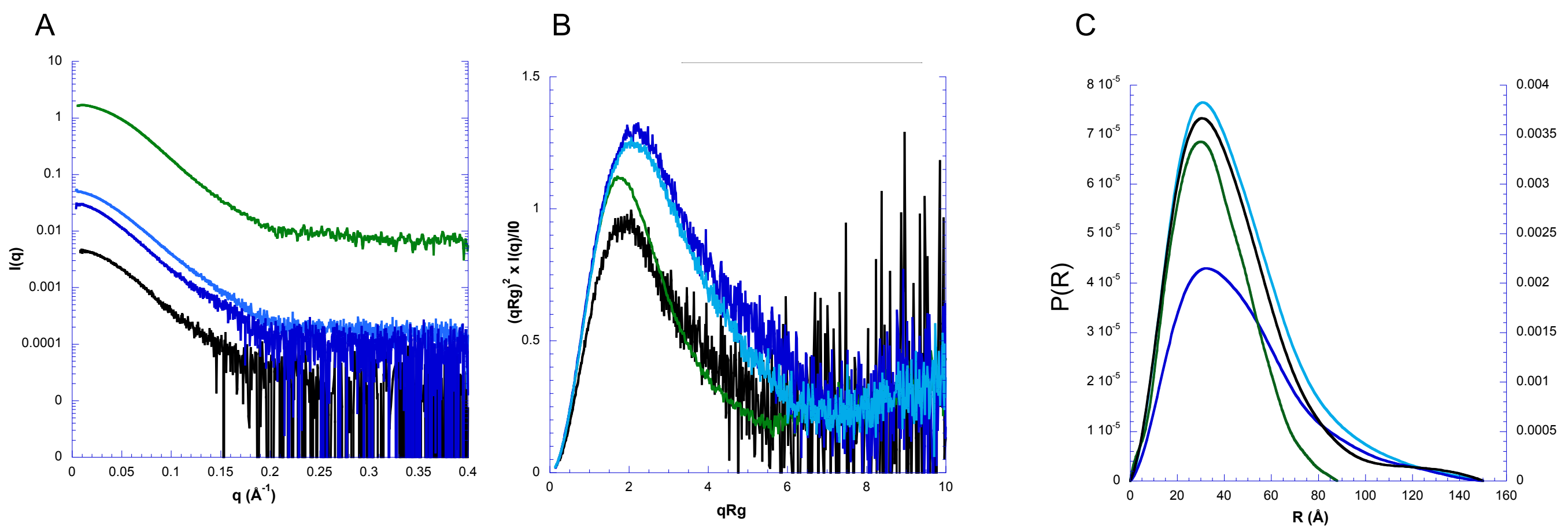

dSuFu hSuFu hSuFu $\Delta 30$ zSuFu 
A

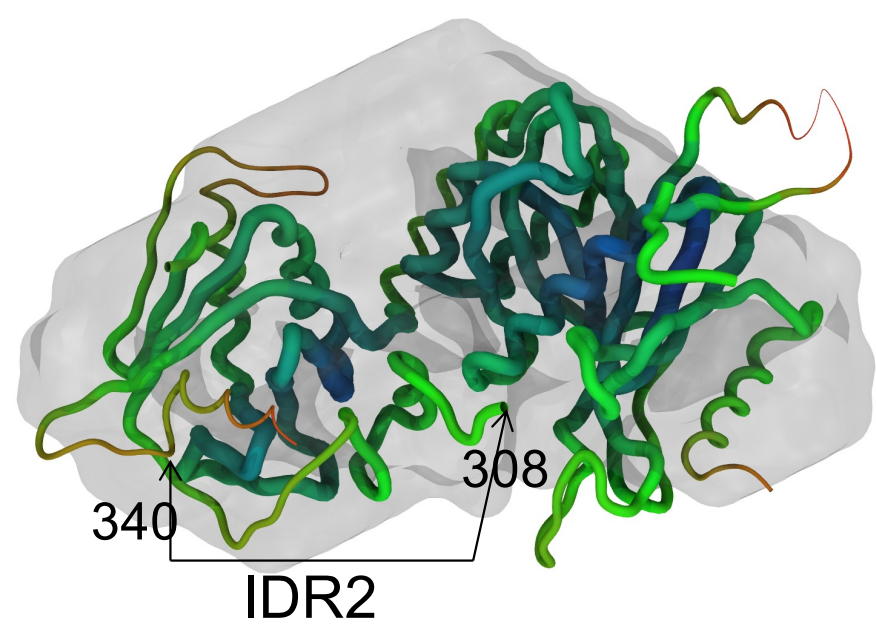

C

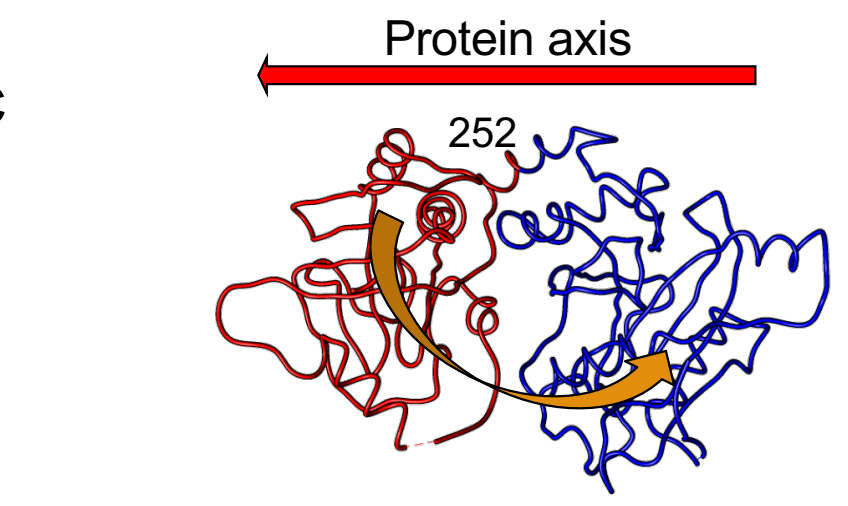

B
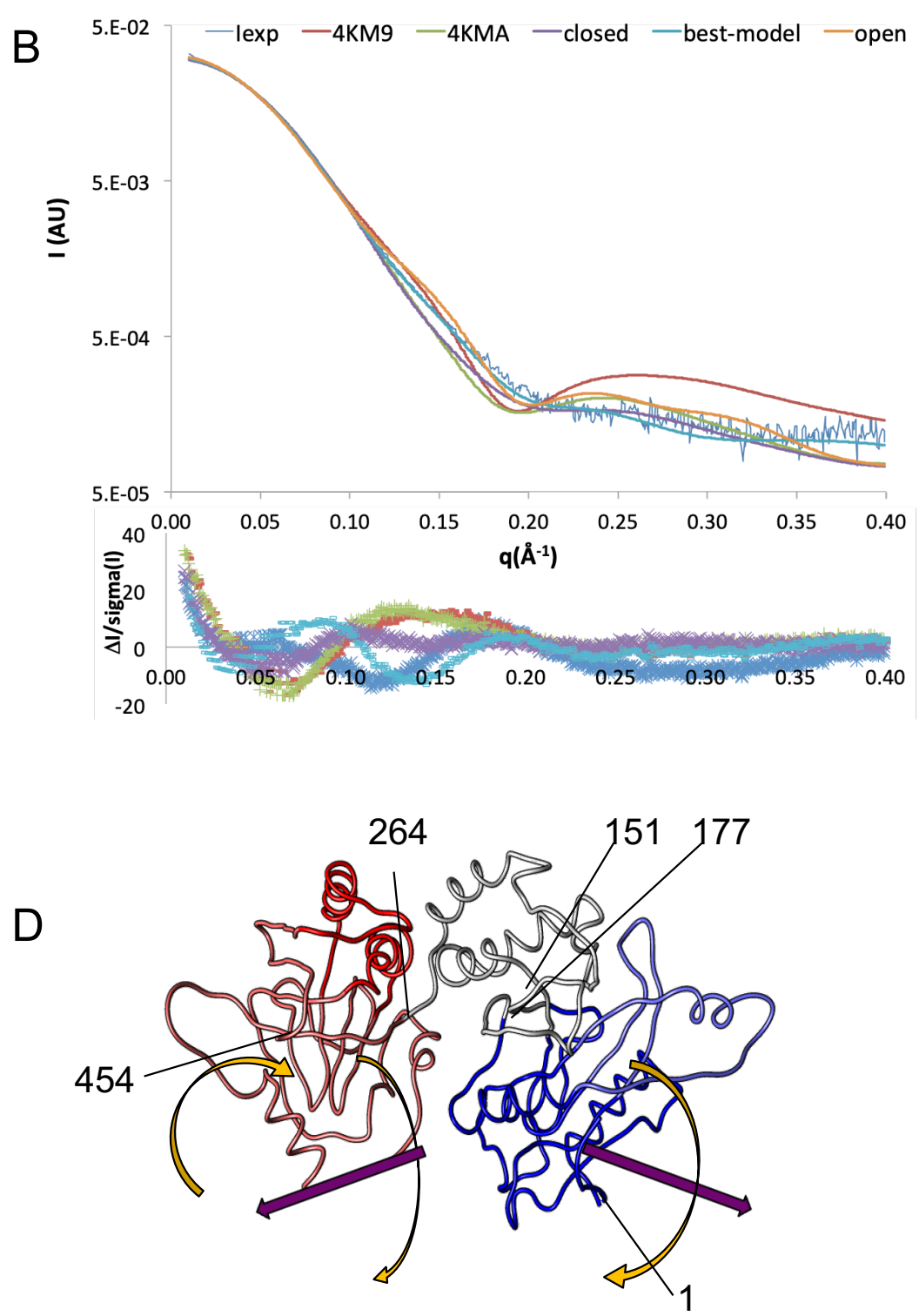

Figure 3 
A

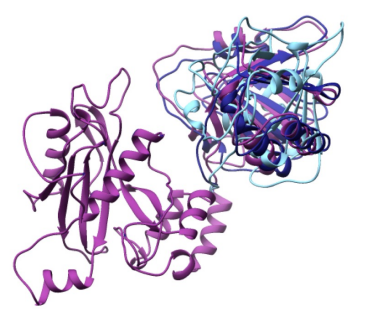

B

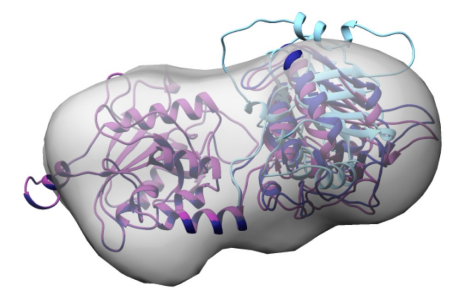

D

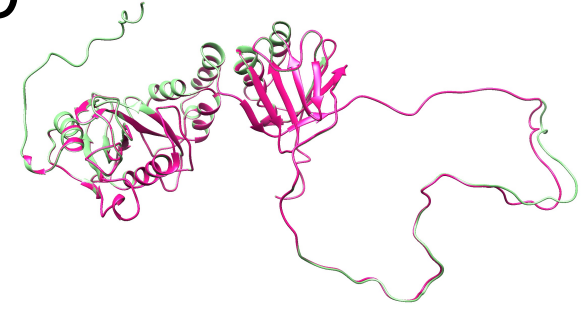

$E$

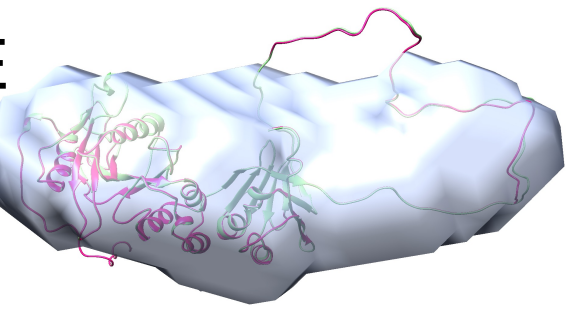

C

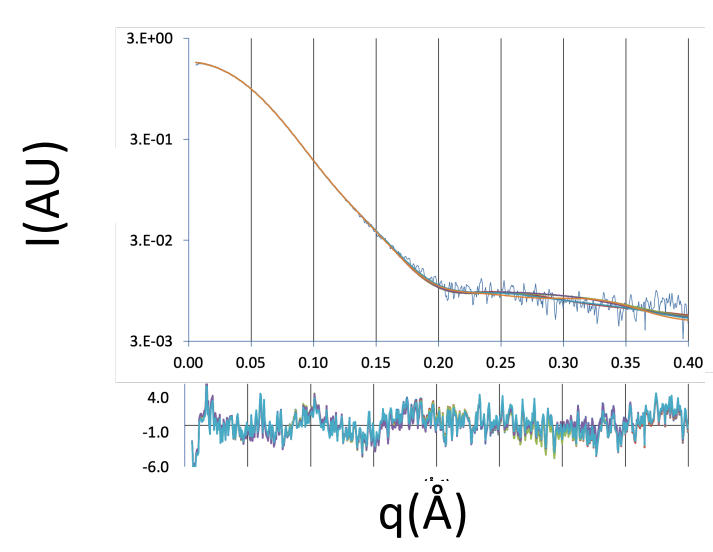

$\mathrm{F}$

$\coprod_{3 . \mathrm{E}-05}^{3 . \mathrm{E}-03}$

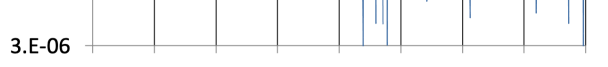
$\begin{array}{lllllllll}0.00 & 0.05 & 0.10 & 0.15 & 0.20 & 0.25 & 0.30 & 0.35 & 0.40\end{array}$ \begin{tabular}{l|l}
5 \\
0
\end{tabular} $q(\AA ̊)$
G

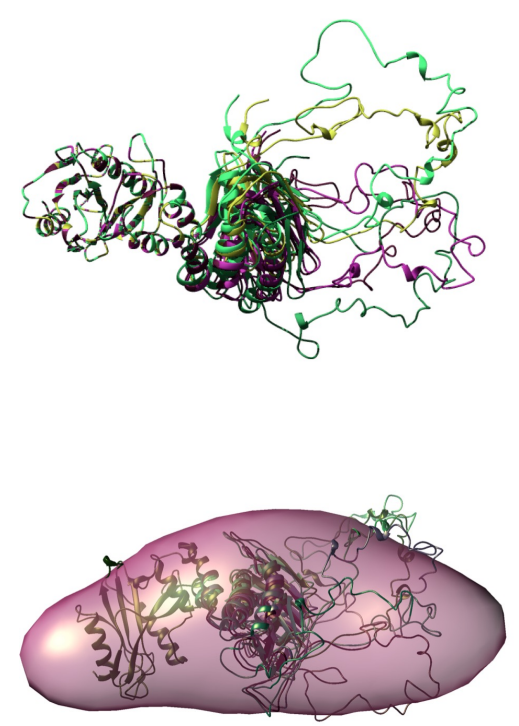

$J$

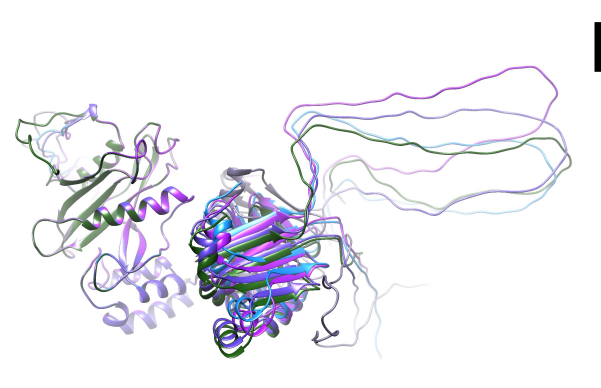

$\mathrm{K}$

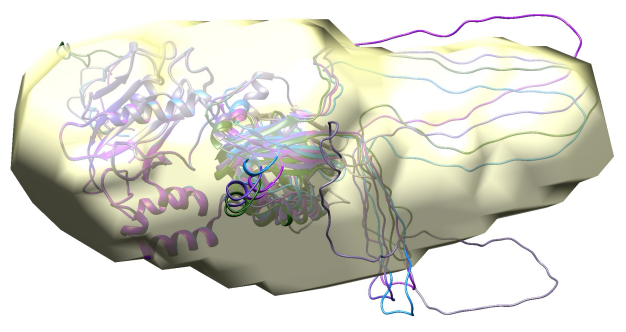

$\mathrm{H}$

L
Figure 4
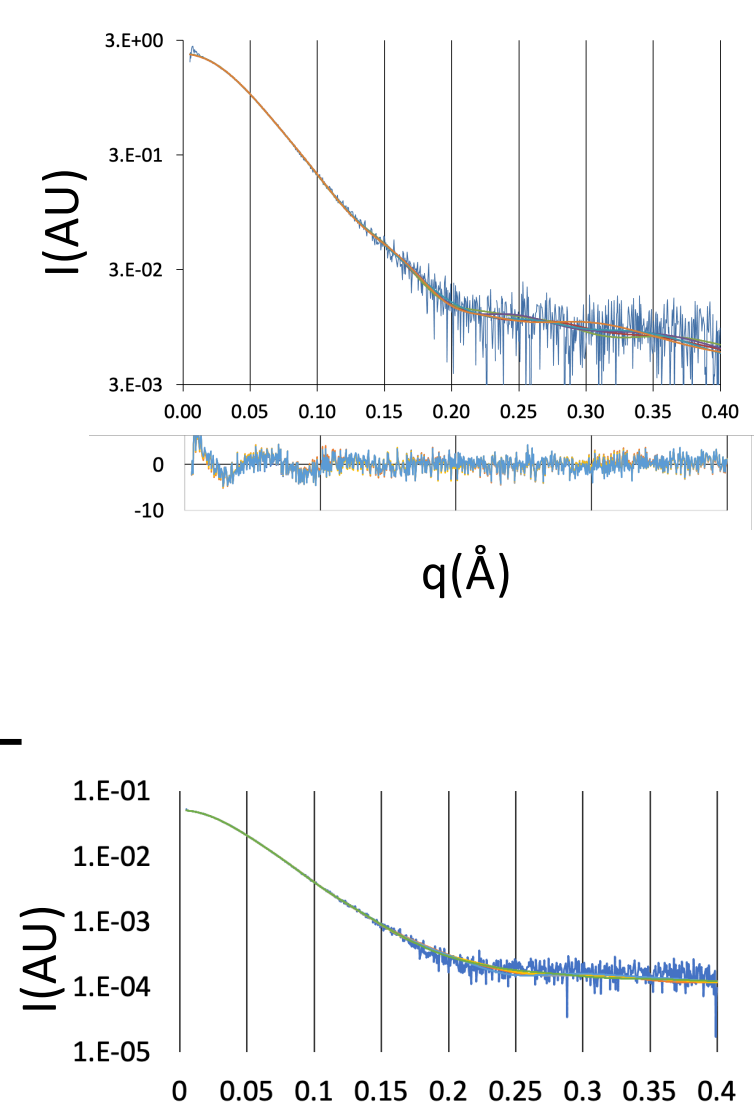

$\begin{array}{llllllll}0.05 & 0.1 & 0.15 & 0.2 & 0.25 & 0.3 & 0.35 & 0.4\end{array}$

2. $q(\AA ̊)$ 
Figure 5
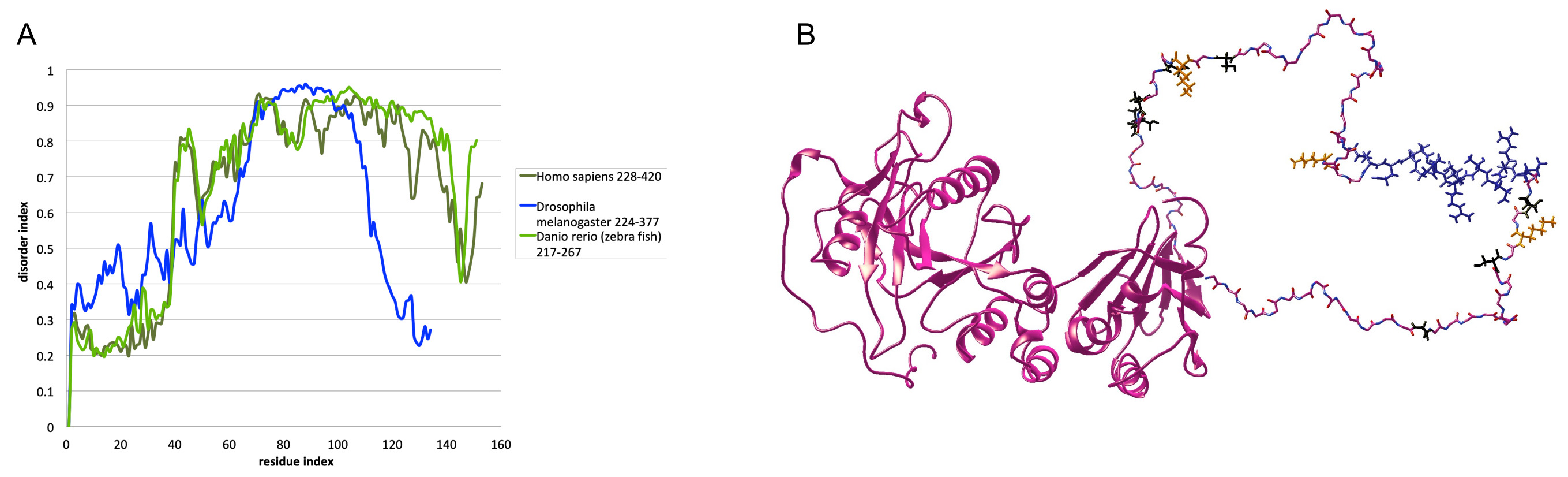